Viewpoint

\section{Novel foods and food ingredients: what is the mission of scientists and technologists?}

\author{
Manuel Mota* and \\ José Empis ${ }^{\dagger}+$
}

*Centro de Eng- Biológica - IBQF, Universidade do Minho, Braga, Portugal

†Centro de Eng- Biológica e Química, Instituto Superior Técnico, Av. Rovisco Pais, P-1049-001

Lisboa, Portugal

(Tel.: + 351-218417889; fax: + 351-218417889; e-mail: pcjempis $@$ popsrv.ist.utl.pt)

\begin{abstract}
The situation concerning world food production is changing dramatically. On the one hand, the world population is expected to grow still further, reaching about 8 billion people in the middle of the 21st century. Novel phenomena, such as ageing of the human population and excess weight in developing countries pose new nutrition problems. Food scares, which were enhanced by recent BSE and toxicological outbreaks, hinder the applicability of some solutions. Under these conditions, scientists and technologists should participate actively in the tasks of informing the population as well as finding new ways to provide people with the optimal amount of nutritious food. (C) 2001 Elsevier Science Ltd. All rights reserved.
\end{abstract}

\section{Introduction}

Food scientists have existed for some time, largely at first because of the social need to fight fraud. This need

†Corresponding author. produced the first breed of food chemists and physicists, mainly working for the regulatory authorities in the 19 th century. As food production transformed itself in keeping with the industrial revolution, food scientists accumulated in industry. As their scientific capabilities and knowledge progressed, they became capable of tackling the extremely complicated biological matrix even without a more or less immediate economical objective, and, therefore, found their niche in universities and research institutions too.

Food scientists are trained, at high cost to the community, because food is recognised to be of an overwhelming importance, from the economic, strategic and political points of view. Nutritionists, mainly involved in public and personal health and quality of life, appeared much for the same reasons.

It is, therefore, probably indisputable that food scientists and nutritionists when taken as a group will master in an unrivalled way the potential, the trends of demand, the physicochemical and nutritional properties and the marketing complexities of novel foods and food ingredients.

We would like to point out, as Science recently did [1], that it is, therefore, their utmost professional duty to society, to participate and indeed to foster, efforts capable of informing the public about the present and foreseeable food-related problems for humanity, about the existing answers to perceived problems, and about the major hurdles and proposed solutions for them. For this purpose, they must train their communication skills by debate, and resolve their scientific differences before addressing the community.

The strong progress in information technologies enabled both an unprecedented data crunching capability for the knowledgeable individual, and a wider, apparently all-embracing awareness by the public in general, largely due to efforts by the media and, of course, the internet. As regards food, this means that in industrial or post-industrial societies, data availability has spread to such an extent that, given any motivation whatsoever, individuals may gather data and construe arguments in favour of any of their beliefs, even if their origin is far from rational.

Novel foods and food ingredients, inasmuch as they represent a breach with tradition, naturally fall into an especially controversial class of eminently publicised and controversial subjects. One should ponder about 
this and decide whether the professional standing of a food scientist enables and dictates he should in some way participate in the controversy surrounding them.

The intervention of food scientists in the process of social progress - especially for the case of genetically modified organisms (GMOs) — was one of the underlying aspects of an extremely important and publicised OCDE conference recently held in Edinburgh [2], which provided many reference position papers and critical analytical views. We believe they will contribute to the process of changing attitudes towards novel foods, and affect both the public and the scientists' actions in the near future.

\section{The quest for healthy, nutritional and satisfying food}

The physical and chemical properties of food constituents, the way they interact and the way they are arranged in the food matrix have a deep influence on the intensity of the functional properties of food, important for its processing, preservation and mouthfeel.

It is not only the concentration of different food components-lipids, carbohydrates, and proteins - that matters when a plurality and choice of food items is available. The arrangement of the various components in the food matrix is also of great importance. Are the carbohydrates concentrated at the food surface? Are they homogeneously scattered throughout the whole food? Are they arranged in discrete aggregates? What is the degree of intimacy between lipids and carbohydrates?

All the above characteristics will ultimately influence several functional properties - crustiness, crispiness and smoothness, colour preservation or resistence to lipid oxidation, but also many other more subtle properties such as flavour persistence in the mouth. In other words, the microstructure plays a key role whenever functional properties are concerned [3]. That microstructure and branching are a key factor in transports phenomena was also raised in cancer cell therapy, where several scientists stress the influence that modified cancer cells and capillary network formation in the vicinity of tumours might have on the efficacy of cancer cell therapy [4-7].

The kinetics of flavour release may not, however, be too slow; or else the food will be chewed and swallowed before the flavour can ever have the chance of being perceived. In other words, diffusion throughout the food matrix will regulate the flavour perception and its persistence. On the other hand, a flavour must be brought to the consumer's mouth with all the characteristics, which were initially introduced in it along with its processing. This means that the food matrix must have a set of properties that allow for the stabilisation of the flavour inside it as long as the food is not consumed.

The retention and the stabilisation capacity of the food product will thus depend not only on the food composition but also on the surface properties of the different food components, especially those that are supposed to be in direct contact with the additive. Thus, specific surface area, hydrophobicity, crystallinity are properties that will have to be studied to anticipate the additive retention capacity by a certain food matrix. To understand the interactions between the food microstructure with its ability to retain and release food flavours, it is necessary to characterise the microstructure, to measure the water retention capacity and the hydrophobicity of each flavour and, last but not the least, to carry out transport studies of the different flavour molecules through the food product and derive appropriate models.

These considerations are examples of practical problems for which food scientists have devised many solutions, and which underlie the usefulness of the concept of food functionality.

Functional foods on the other hand, must contain significant levels of biologically active components that impart health benefits or desirable physiological effects beyond basic nutrition, and which may be present or absent as a result of technological or biotechnological means. Thus whilst ensuring an appropriate rate of flavour release is a feat attributable to the functional properties and microstructure of food, the fact that eating it will induce a pleasurable sensation including endorphin release, with a plausible contribution to physiological wellbeing, might be invoked in naming that food as functional.

A more realistic example is probiotic food [8]. Probiotics may be included in functional foods, which are food components that are microbial and improve gastro-intestinal balance and add digestive enzymes. In general, they help maintaining an overall state of wellbeing and body fitness, normally by enhancing the natural defences of the human organism. High adhesion to gut epithelium has been proposed as involved in the mechanism of probiotic action [9]. Other works suggest that probiotics may be involved in the stimulation of, or may be used to regulate, the immune system and the problem of food allergy [10-13]. It was proven that probiotic action could be substantially enhanced with the addition of appropriate carbohydrates, which promote the fixation of the probiotic population, thus acting as prebiotics [14]. A typical example is inulin, a polysaccharide contained in many vegetables and fruitschicory, Jerusalem artichoke, bananas, among many others - which promotes the fixation of certain beneficial lactic acid bacteria in the intestinal gut [15]. Another example is chitosan, a polysacharide that stimulates vitamin E uptake [16], but could have been added for its functional properties. The investigation of many carbohydrates and their effect on promoting probiotic action is an actual topic [17], and much remains to be done. Other research needs include more methods to be developed to characterise intestinal microflora, including the non-culturable species, the understanding of the role of the diet and probiotics, and viable or non-viable cell wall components in the general immune functions [18]. 
Functional foods are still food, and should be considered so by their producer by the consumer and by the regulatory authorities. Industrial associations have successfully undertaken to negotiate with the authorities a "Code of Good Practice for Health-related Product Claims" in many European countries. Their development and popularity is a milestone in the bridge from food research to health.

Another category of products, which might include food supplements, are nutraceuticals, which claim to have health benefits, including the prevention and treatment of disease, but no longer should be considered as food. Examples of nutraceuticals include amino acids, fats (modified or not), dietary fibres, plant pigments, antioxidants, vitamins and minerals such as calcium or iron. Some sweeteners may be termed nutraceuticals, e.g. for diabetics.

Recently, experiments on the genetic expression of vaccines in vegetables or fruits, such as bananas or potatoes have been reported, which may allow low cost distribution of some of these "medicinal foods" [19]. If this concept is widespread, and if the concerned population accepts the consumption of these types of food, then the range of nutraceuticals utilisation will be considerably enlarged. Such nutraceutical products are the last milestones bridging food and pharmacy. These foods may be especially important for groups of consumers with special nutritional deficiencies, even in industrial societies, as pointed out by Dr. Weksler [2], who lists nutritional deficiency in protein, vitamins $\mathrm{C}$, $\mathrm{A}, \mathrm{B} 6, \mathrm{E}$, and $\mathrm{Fe} / \mathrm{Zn}$ in a significant proportion of the US senior population.

We believe to have therefore summarised actual trends in food research in three areas which have relatively recently emerged as autonomous, functional properties and food microstructure, functional foods, and nutraceuticals, in that order. Much remains to be done in all of them as well as in the processing aspects of food production and preservation, in order to maintain the functionalities, functional food properties and their nutraceutical activities.

\section{A perspective on food-related problems}

When trying to make sense of a collection of disperse, important, and actual facts, it is difficult to show impartiality. Historians claim there is not enough distance to create an unbiased perspective. Biased as the authors may be, the possible perspective could perhaps best be imagined as that perceived by an imaginary and clairvoyant observer of Planet Earth.

This being would rapidly conclude this planet is inhabited by a dominating species, which calls itself the human species. This race had shaped the planet in order to adapt it to its existence and proliferation. It has mastered the methodologies for water usage and food production and preservation in a large part of the inhabited areas. A more detailed analysis would then lead him to the conclusion that some more disturbing and real facts persisted, namely:

(a) That whereas food surpluses consistently exist in some parts of the planet, food shortages consistently appear in some others. These food shortages cannot be exclusively explained by climatic disasters or by inappropriate land. Factors such as generalised underdevelopment, low education, illconditioned foods, inadequate food distribution and-preservation facilities all contribute to them. This present situation might well be menaced by world population growth, which might attain 8 billion by mid 21 st century.

(b) That no great progress had been gained, or was expectable [2], in redistributing excess food to hunger stricken areas, even if regional surpluses had to be diverted to non-food applications.

(c) That those over-consumption patterns were apparent in affluent areas leading to a series of characteristic diseases, and malnutrition or even hunger was obvious in others. It must be noted that an emerging and apparently contradictory phenomenon is appearing in developing countries, where a starving population coexists with an evergrowing overweight population (in Brazil and in Colombia the percent overweight population reached in 1999, 36 and $41 \%$, respectively [20]). This raises the question of how well the glucose and lipid metabolism is known. It is nowadays almost certain that different people evidence significant differences towards fasting tolerance, for example. It is of general consensus that genetic reasons are linked to steatopygia - a morphological trait related with fat accumulation on the buttocks of pearshaped women - which corresponds in itself to a marked resistance to prolonged starvation. This might explain - for merely selective reasons - its significant incidence in developing countries, where body-fat accumulation could bring about a competitive advantage for successful pregnancies during times of famine. The group of steatopygous women are prone to quick lipid accumulation and, quite likely, they will show modified glucose metabolism. Accordingly, this group will also be prone to overweight in times of food abundance.

(d) That it was exactly in the less developed areas, where food was scarcest, that minimal productivities were achieved, as well as maximal inefficiencies leading to wastage in the production and preservation of food.

(e) That this wastage amounted to an important part of overall production, hence that overall production was consistently planned for an amount sufficient to compensate for it. 
(f) That in areas of efficient production, accumulation of residues was consistently growing, resulting in the accumulation of those agricultural inputs which did not degrade at appreciable rates. Productivity increase in industrialised agricultural systems was nevertheless becoming marginal, in comparison with past successes, suggesting that maximal productivity had been attained, or nearly so, with existing cultivars and arable land [2].

(g) That some of the factors determining quality of life were suffering from this intensive production practice. If the clairvoyant being also had the possibility to examine historical development and was knowledgeable about population trends, he would not be able to miss the staggering increase in human population brought about by, among others, the invention of fertilisers and antibiotics, which permit the survival of a larger, and longer living population, respectively. Productivity gains largely stemming from seed and agricultural procedure improvement were showing unmistakable weakening [2], and global, future food shortage a more real menace than might have been expected.

(h) That despite strong progress in food production and preservation technologies, and the ever growing importance of good manufacturing practice, even in the more affluent areas there were many examples of situations where malpractice or plain carelessness had actually lead to the inclusion of toxic components in food, and to situations in which it became expectable that food-borne disease might develop. In these regions, there were operational regulatory institutions, whose mission was to ascertain that food quality be maintained and that food items of actual or potential toxicity not be consumed. Nevertheless, they were powerless to predict and prevent them, proving that new views, new studies and new methods were needed in order to improve the knowledge and the control of upcoming outbreaks.

(i) That extremely contagious "food scares" were becoming prominent, at a pace more or less exactly matching that of increased food safety, and that these were either poorly correlated or actually unrelated to any proven toxicity in some of the particular foods they were all about, and mainly due to malpractice.

(j) That at the same time, mainly due to changes in ways of life, consumer preferences, and technological progress, the scientific, mainly the medical community, was well aware of existing problems in the food supply, namely because the selection pressures had become favourable to opportunistic psychrotrophs in minimally processed food.

(k) That a topic to which special attention was being paid by the media, and had somehow become an international concern, was that of GMOs, when used in food products, despite the fact that experimental evidence had accumulated as to their innocuity, were being pointed out as potentially dangerous.

(1) That as a consequence of these concerns, laws were published in some countries banning the use of GMO, and rendering agricultural production systems more, rather than less different from those used in otherwise similar countries. This happened after a first round of GM food crops, arguably more beneficial to producers than to consumers, was developed, but those laws introduced regional asymmetries, discouraging research and nurturing production disadvantages.

(m) That the scientific community had not assumed per se and as such any important role, other than developing the GMOs.

(n) However, he would also notice that three unrelated and very recent events might be cornerstone to a change in public perception of the risk eventually associated with GMO food:

- the creation of a "Council for Biotech Information", announced in Washington 3 April and initially endowed with US $\$ 50 \mathrm{M}$, by a coalition of seven leading companies with an interest in biotechnology, plus the industry trade association - the Biotechnology Industry Organisation - and whose purpose it would be to foster understanding of biotech by the US and Canadian Public shows how important this issue is;

- the creation of a "European Food and Health Authority", (European Commission, 1999) depending from the Directorate General for Consumer Interests.

- the likely event that a liability clause be explicitly included in the marketing authorisations of GMO foods, for the admittedly unlikely event of damages ocurring from their production and consumption.

(o) Last but not least, the EU Commission has undertaken to restore confidence in the usage of GMOs in food, after recognising that the actual situation is hindering research and competitivity in Europe.

\section{GMOs in food production}

Having thus characterised the present situation as seen by the imaginary clairvoyant observer, it is important to elaborate in first place about GMOs, for they are the item which bears a closer relationship to recent scientific progress.

There are two attitudes concerning GMOs. The first one is that of general acceptance, whenever use of 
GMOs is shown to be visibly advantageous for health care. Thus, genetic medicine technologies, which effectively change, for instance, the enzyme producing capability of individuals, are currently in use. On the other hand, no one has questioned the use of insulin made from GMOs. Quoting Roller and Harlander [21], "Many products of biotechnology which are acceptable in the treatment of terminal disease are not favoured when applied to food. When biotechnology is applied to food, consumers have a choice of whether to accept the technology or not. The exercise of informed choice requires information, and the provision of and reaction to such information will be a key factor in the acceptance or otherwise of food applications of biotechnology".

We may thus anticipate that a positive attitude will be observed when genetically modified tobacco plants will be extensively cultivated for the production of haemoglobin [22], or, as is proposed in a very recent work [23], for the production of human somatotropin.

The recent development by Bizily and co-workers [24], who genetically modified Arabidopsis thaliana in order to get it to scoop out mercury from contaminated soils, a problem which conventional technologies a cannot solve economically, might well prove to be a landmark in altering this attitude. It is also expectable that the general public attitude towards GMOs in food will change when vegetables adapted to harsh growing conditions, and which undoubtedly contribute to the increase in food availability - resistance to drought, to soil with high salt content, or to extreme temperatureswill be opened to agricultural exploitation [25].

The second attitude concerns the use of GMOs or their derived products as food staples. It seems that the public attitude is at least of caution and concern. This attitude is especially visible in European countries, and, from our point of view, must not be dissociated from the recent events in the Food and Feed areas: BSE, Listeria and E. coli outbreaks with lethal consequences, may be in part responsible for this attitude.

Scientists whose knowledge represented a heavy societal investment have the possibility, and therefore the mission to tell the public some simple facts. Apparently, very few people are aware that they eat transgenic material whenever they eat anything, except if their menus are exclusively cannibal. Few people are sufficiently aware that the digestive system breaks down foodstuffs to small pieces in an extremely effective way, including not only proteins, lipids and carbohydrates, but also foreign DNA, whatever its origin. It appears that ingestion of foreign DNA does not pose a risk [2]. Indeed, even the probability of ampicillin resistance gene being transferred to competent E.coli has been shown to be less than $1.7 \times 10^{-20}$, or 60 million times less probable than finding at least one Clostridium in a high $\mathrm{pH}$ canned food.

Few people are aware that allergenicity to food is inevitable and that there are people allergic to such current foods as onion, garlic, cheese, strawberries, different kinds of nut, seafood and even whitefish, to hen eggs but not to quail's, to cow's milk but not to goat's, and to proteins containing phenyl-alanine.

It is therefore to be anticipated that some, few, people will undoubtedly be allergic to GMO foods, as pointed out by Nordlee [26] for a transgenic soybean, but the vast majority of the world population will safely consume GMOs that have successfully passed all the regulatory tests that were devised whenever a new food product is intended to be marketed. The conclusion of a panel who undertook an assessment of genetically modified soybean with an inserted gene, was that the bean and its products were comparable to, and as safe for human consumption as, conventional soybean and products obtained from it [2].

In any case, the transfer of genes conferring resistance to drought and to high salinity into cash crops must evoke reasonably general approval. Together with other, second-generation GM seeds providing measurable advantages to the consumer as well as to the producer, they will probably contribute to build up a positive public opinion, eventually capable of self-expression too.

\section{Food-related shocks}

That food scares indistinctly target some of the best and most powerful developments in food production and preservation and some of the cheapest and most vile examples of malpractice or carelessness alike, often likening the way they are perceived by the public, is something food scientists should really worry about. This situation probably originates because of misinformation or insufficient information of the media and consumers alike, about the taxing and careful regulatory procedures for novel foods. It also implicates some degree of implicit mistrust in these procedures.

It is known nowadays that food-poisoning outbreaks may sometimes come from unexpected micro organisms which for centuries cohabited harmlessly with man and as such have not been paid much attention to by food scientists (e.g. Acinetobacter is now suspected of being the responsible bovine spongiphorm encephalopathy [27]). The presence of pathogenic micro-organisms in unsuspected places and conditions should be carefully monitored, as was recently exemplified by findings of possible cross-contamination of carcasses by $E$. coli O157:H7 in supposedly hygienic North-American abattoirs [28]. Changes in food consumer's habits, changes in the processing methodologies, changes in the food storage procedures may be included, among others less known, as possible causes for this emerging situation.

On the other hand, psycrotrophs such as Yersinia enterocolitica, Escherischia coli and Listeria monocitogenes, some pathological strains of which have been implied, respectively, in gastro-enteritis with a plausible link to arthritis [29], in deadly food poisoning incidents, 
and in gastro-enteritis, miscarriage and a wealth of other diseases, have received widely differing coverage in the literature provided by food scientists. The first paper on Yersinia behaviour towards food processing was published in 1995 [30], and only a handful was published in 1999, whereas a wealth of literature is available for the two others, mainly because of the intensity of the food scares associated with them. But irradiation treatment, which at low or intermediate dosage is efficient against these, has not become more popular or widely accepted.

Another striking example of a dangerous pathogen is Helicobacter pylori. Recognised as being one of the main causes of peptic ulcers [31], its relation with gastric cancer seems plausible [32], but the food microbiology of this organism has not to our knowledge been reported on in depth, though it is tentatively concluded that good manufacturing practice is connected with a low incidence of the $H$. pylori infection [33].

\section{EU legislation}

Novel foods and food ingredients are defined in the EU Directive 258/97. These products may belong to one of six different classes according to the classification set forth in the joint Parliament and Council ruling 258/97, and legalisation procedures for bringing them to market have also been implemented, by way of the Commission recommendation number 97/818. These procedures involve in some cases relatively detailed toxicology studies, but rely on the concept of "sufficient similarity", when the novel item is similar enough, and not expected to involve a higher rate of consumption than another, non-novel, established food item.

In parallel, for food containing or produced from GMOs (recently specified at levels of inclusion at or above $1 \%$, in Commission Regulations (EC) 49 and 50/2000, in force from today onwards, specifically for those already authorised for food use in the EU, mention must be made of this fact in a label (Council ruling 1139/98).

These laws eventually remit to the Council Directive $90 / 220$, which states the procedure for authorisation of cultivation in the EU of GMO seeds. Based on this environmental directive no new authorisations have been forthcoming since 1998, though quite a few are pending, and the existing authorisations are suspended.

\section{Analytical aspects of novel foods}

Novel foods may present challenging analytical problems, as they are in many cases similar to the food items they may substitute. Thus it is indeed difficult in many instances to determine:

(a) Whether a food contains or consists of GMO material, even if such a material was used in its preparation.

(b) Whether a food contains or consists of a fraction of some GMO material which because of its pro- cessing does not appreciably contain protein or nucleic acid fractions.

(c) Whether a food has been subjected to a process operation which substantially changed the nature of chemical species present, even if only that of a minor fraction of these, as might be the case after $\gamma$-irradiation, ultrasound or extreme shear stress processing, all of which are known to result in the production of free-radical species, or indeed by high pressure processing, which affects macromolecular structure.

These analytical problems arose and still arise because of the pro-active attitude the EU, and some European countries on their own initiative, have taken in order to efficiently monitor their food supply, an attitude which will in all probability result in the establishment of a central Food Authority for the EU.

Irradiated foods, though probably not very novel and indeed falling under autonomous legislation, are certainly a good case study because of the public concern they apparently generated as well as because of the intended benefits their use entails.

Detecting previous irradiation of foods proved to be more of a problem than initially anticipated, and also than might be expected if one were to take some of the more apprehensive press releases about it at face value. After an extensive, long lasting and multinational research programme established wholesomeness of any food irradiated with up to $10 \mathrm{kGy}$ mean dose, it took another concerted effort sponsored by the BCR (Bureau communautaire de Réference), now SMT (Standards, Measurement and Testing) activity within the Growth programme of the European Union DG Research to screen potentially useful analytical methodology, followed by standardisation work performed by the working group of the Centre Européen de Normalisation-CEN TC 275 WG 8 - to establish standard analytical procedures. It is noteworthy to mention that the majority of these rely largely on alterations suffered by indigestible components in food (bones, cellulose, silicates, hydrocarbons) and only one relies on the detection of minute amounts of supposedly radiation specific compounds derived from its fatty components, the 2-alkylcyclobutanones. Changes in viable micro-flora and in food macromolecules can also be apparent, but may not in general be used for more than screening tests, and may in some instances arise due to other process operations.

Detection of the use of GMOs in food can be even more of an esoteric endeavour. The two different types of methodologies now undergoing standardisation within CEN TC 275 WG 11 are based on the detection of altered nucleic acid fragments and peptide material. Though the sensitivity of nucleic acid detection methods, largely because of the exponential amplification granted 
by the polymerase chain reaction (PCR) techniques used is theoretically limited by the presence of at least only one such fragment in the initial sample, and in present day practice by perhaps 20 of those, it must be understood that the nature of these fragments must be characteristic of the genetic modification, or modifications, whose existence is being analysed. It is thus usual to look not for the altered nucleic acid per se, but instead for the promoter or terminator sequences which were used for its effective inclusion. This specificity of the PCR based methods means that a multiplicity of such tests must exist, capable of detecting one characteristic sequence each. It also means that their effectiveness is conditioned by advance knowledge of the target sequences' presence. It ultimately depends, therefore, on a statement from the producer of the GMO containing information on precisely those target sequences: This information has, as far as we know, always been available. But the sheer complexity of the analytical methodology involved in looking for GMO nucleic acid sequences without prior knowledge renders their detection implausible in such a scenario. The situation is therefore characterised by the fact that successful detection of GMO material depends on their developers' conformity to regulations, which might well be unenforceable. Therefore, it is not inconceivable that biological materials might have been developed already, and are being marketed as non-modified, using promoters and terminators which are not foreseably traceable.

Even the presence of genetically modified material for which the necessary primers are available may be hindered if the food has been processed. Thus it may be impossible to detect whether a vegetable oil is a blend containing oil from GMO seeds, even if the detection of the appropriate PCR method is fantastically low and the EU threshold for declaration is $\geq 1 \%$, because the operations involved in edible oil extraction and processing effectively removed all nucleic acid or maimed any residual fragments beyond PCR recognition. Exactly the same may apply to some processed ready to eat food items, such as cakes or cooked meals, when the processing has had the same effect.

The other methods for GMO detection use immunological detection of peptide components characteristic of the particular GMO, which is being traced. Much the same limitations exist as for the nucleic acid methods, i.e. one has to know what one is looking for and have the necessary antibodies, and one must be aware that protein denaturation throughout the processing chain will render detection implausible. The "natural" detection limits in immunological methods are powerfully low, when compared with the $\geq 1 \%$ EU legal threshold, but this applies only to relatively non denatured material, and, therefore, many food items which contain denatured protein also will remain undetected.
In any case there will in all probability be readied for September 2001 at least two provisional European standards for GMO detection. And there are of course national standard analytical methods in various European countries.

\section{Conclusions and perspectives}

At the end of the OECD Edinburgh conference on GM Food Safety: Facts, Uncertainties and Assessment (28 February-1 March, 2000) [2] the need for a more open, transparent and inclusive debate and for openness and transparency in the policy process, as well as an acknowledgement that there is potential benefit to be gained from GMO technology, together with the need for long term toxicity studies in humans, and a debate on the trade consequences of GMO was recognised.

The European Community presented recently a "White Paper on Food Safety" [34], which, essentially as a result of an earlier report by an international group of consultants [35], advocates the creation of a new Food Authority which might provide to the European consumers some reassurance about the efficiency of regulatory measures in force.

This is badly needed from a trade point of view. Very recent data from the US seems to show that, contrary to earlier expectations due to press releases from people or groups with an active anti-GMO stance, plantings of GMO material were meeting producers' expectations in the US. This means we will go on importing the permitted GMO for use in feed and food and our farmers will still not be able to compete against these seeds with similar material. This situation, whilst certainly providing data for long term toxicity studies of populations relying on heavy imports of GMO, for instance for feed, as is the case of Portugal, certainly needs redressing.

But even with an impartial and knowledgeable Food Authority in action, and it will take some time, there is no substitute for debate, preferably publicly held. As states Dupré [36], "To make an informed decision about whether to proceed with genetic modification, one should be able to call on an equally thorough investigation of the consequences of the alternatives, such as organic farming. Other complex economic and social questions must be answered as well. What new skills will be needed in the labour pool? What are the implications of genetically modified foods for the existing transportation and distribution methods?"

Scientists should thus prepare, intervene, research and transmit to the coming generation what they believe to be our perceived needs.

Meanwhile, acreage of GMOs will undoubtedly increase due to their superior characteristics and increased availability, even if mainly in the developing countries. And the extremely probable absence of 
deleterious effects on the populations consuming those crops will eventually convince those which presently advocate the imperious need for long term toxicological studies that they were too cautious. And GMOs will have helped mankind cope with ever decreasing ratio of arable land per inhabitant, whilst becoming ever more important for the availability of novel consumer-adapted foods and ingredients.

\section{References}

1 Farmelo, G. (2000) 'Why Should Scientists Bother Talking to the Public?' in Science, 7 January.

2 OECD Edinburgh Congress (http://www.oecd.org/subject/ biotech/ed_prog_sum.htm) European Patent DE19845246, 1999

3 Mota, M., Teixeira, J.A. and Yelshin, A. (1999) 'Image Analysis of Packed Beds of Spherical Particles' in Separation and Purification Technology 15, 59-68

4 Gottlieb, M.E. (1991) 'Vascular Networks: Fractal Anatomies from Non-linear Physiologies' in IEEE Eng. Med. Bio. Mag. 13, 2196-2197

5 Nekka, F., Kyriacos, C., Kerrigan, C. and Cartilier, L. (1996) 'A Model of Growing Vascular Structures' in Bull., Math. Biol. 58, 409-424

6 Chaplain, M.A.J. and Anderson, A.R.A. (1997) 'Mathematical Modelling, Simulation and Prediction of Tumour-induced Angiogenesis' in Invasion and Metastases 16, 222-234

7 Heckman, C.A. and Jamasbi, R.J. (1999) 'Describing Shape Dynamics in Transformed Cells Through Latent Factors' in Exp. Cell. Res. 246, 69-82

8 Kalantzopoulos, G. (1997) 'Fermented Products with Probiotic Qualities' in Anaerobe 3, 185-190

9 Bibiloni, R., Pérez and De Antoni, P.F. (1999) 'Will a High Adhering Capacity in a Probiotic Strain Guarantee Exclusion of Pathogens from Intestinal Epithelia?' in Anaerobe 5, 519524

10 Romond, M.B., Haddou, Z., Mielcareck, C. and Romond, C. (1997) 'Bifidobacteria and Human Health: Regulatory Effect of Indigenous Bifidobacteria on Escherichia coli Intestinal Colonization' in Anaerobe 3, 131-136

11 Wagner, R.D., Pierson, C., Warner, T., Dohnalek, M., Farmer, J., Roberts, L., Hilty, M. and Balish, E. (1997) 'Biotherapeutic Effects of Probiotic Bacteria on Candidiasis in Immunodeficient Mice' in Infect. Immun. 65, 4165-4172

12 Wagner, R.D., Warner, T., Roberts, L., Farmer, J. and Balish, E. (1997) 'Colonisation of Congenitally Immunodeficient Mice with Probiotic Bacteria' in Infect. Immun. 65, 3345-3351

13 Majamaa, H. and Isolauri, E. (1997) 'Probiotics: a Novel Approach in the Management of Food Allergy' in Allergy Clin. Immunol. 99, 179-185

14 Michel, C., Kravtchenko, T.P., David, A., Gueneau, S., Kozlowski, F. and Cherbut, C. (1998) 'In vitro Prebiotic Effects of Acacia Gums onto the Human Intestinal Microbiota Depends on Both Botanical Origin and Environmental $\mathrm{pH}^{\prime}$ in Anaerobe 4, 257-266

15 Cho, S.S., Prosky, L. and Dreher, M. (eds). (1999) 'Complex Carbohydrates in Food'. Marcel Dekker Inc, NY

16 European Patent DE19845246, 1999

17 Kitazawa, H., Ishii, Y., Uemura, J., Kawai, Y., Saito, T., Kaneko, T., Noda, K. and Itoh, T. (2000) 'Augmentation of Macrophage
Functions by an Extracellular Phosphopolysaccharide from L. delbrueckii spp. bulgaricus' in Food Microbiol. 17, 109118

18 Salminen, S., Ouwehand, A., Benno, Y. and Lee, Y.K. (1999) 'Probiotics: How Should they be Defined?' in Trends Food Sci. Technol. 10, 107-110

19 Prakash, C.S. (1996) 'Edible Vaccines and Antibody Producing Plants' in Biotechnol. and Development Monitor 27, 1013

20 Gardner, G. and Halweil, B. (2000) 'Underfed and Overfed. The Global Epidemic Malnutrition'. Worldwatch Paper 150, March

21 Roller, S. and Harlander, S. (1998) 'Modern Food Biotechnology: Overview of Key Issues', in Genetic Modification in the Food Industry, (Roller, S., Harlander, S., eds, Blackie Academic and Professional, Chapman \& Hall

22 Pagnier, J., Marden, M. and Poyart, C. (1996) 'Current status of haemoglobin-based blood substitutes' in M. S. Medecine Sciences 12, 1342-1350

23 Staub, J.M., Garcia, B., Graves, J., Hajdukiewicz, P.T.J., Hunter, P., Narender, N., Paradkar, V., Schlittler, M., Carroll, J.A., Spatola, L., Ward, D., Ye, G. and Russell, D. (2000) 'High-yield production of a human therapeutic protein in tobacco chloroplasts' in Nature Biotecnol. 18, 333-337

24 Bizily, S.P., et al. (2000) 'Phytodetoxification of hazardous organomercurials by genetically engineered plants' in Nature Biotechnology 18, 213-217

25 'Genetically Modified Organisms (GMOs) - a Backgrounder by the IFT' (2000) Food Technology 54(1), 42-45

26 Nordlee, J.A., Taylor, S.L.J.A., Townsend, L.A.T. and Bush, R.K. (1996) 'Identification of Brazil-nut Allergen in Transgenic Soybeans' in New England Journal of Medicine 334, 688692

27 Tiwana, H., Wilson, C., Pirt, J., Cartmell, W. and Ebringer, A. (2000) 'Autoantibodies to brain components and antibodies to Acinetobacter calcoaceticus are present in bovine spongiphorm encephalopathy' in Inf. Immun. 67, 6591-6595

28 Mackenzie, D. (2000) 'Packed with Danger. Lethal Bacteria Could be Lurking in Meat-packaging Plants' in New Scientist 165(2229), 12

29 Gripenberg-Lerche, C., Skurnik, M. and Toivanen, P. (1995) 'Role of YadA-mediated collagen binding in arthritogenicity of Yersinia enterocolitica serotype O:8: experimental studies with rats' in Infect. Immun. 63, 3222-3226

30 Greer, G.G., Gill, C.O. and Dilts, B.D. (1995) 'Predicting the Aerobic Growth of Yersinia enterocolitica on Pork Fat and Muscle Tissues' in Food Microbiol. 12, 463-469

31 Northfield, T.C., Mendall, M. and Goggin, P.M. (1993) 'Helicobacter pylori Infection: Pathophysiology, Epidemiology and Management', Kluwer Academic, Amsterdam

32 Harris, R., Owens, D.K., Witherell, H. and Parsonnet, J. (1999) 'Helicobacter pylori and Gastric Cancer: What are the Benefits of Screening only for the CagA phenotype of $H$. pylori?' in Helicobacter 4, 69-76

33 Dorta, G., Jornod, P., Blum, A.L., Grehn, M. and Grassner, M. (1999) 'Low Prevalence of Helicobacter pylori Infection in Swiss Adolescents' in Helicobacter 4, 67

34 European Commission (1999) White Paper on Food Safety (http://europa.eu.int/eur-lex/en/com/pdf/1999/ com1999_0719en01.pdf)

35 James, P, Kemper, F., Pascal, G. (1999) 'A European Food and Public Health Authority - The Future of Scientific Advice in the EU', personal copy

36 Dupré, J. (2000) 'The Fight for Science and Reason' in The Sciences $40,40-45$ 